Archives de sciences sociales des religions

118 | avril - juin 2002

Varia

\title{
Nouvelles religiosités argentines
}

Maïra Muchnik

\section{OpenEdition}

Journals

Édition électronique

URL : http://journals.openedition.org/assr/217

DOI : 10.4000/assr.217

ISSN : $1777-5825$

Éditeur

Éditions de l'EHESS

Édition imprimée

Date de publication : 1 avril 2002

Pagination : 71-84

ISBN : 2-222-96718-X

ISSN : 0335-5985

Référence électronique

Maïra Muchnik, « Nouvelles religiosités argentines », Archives de sciences sociales des religions [En ligne], 118 | avril - juin 2002, mis en ligne le 03 juin 2005, consulté le 21 avril 2019. URL : http:// journals.openedition.org/assr/217; DOI : 10.4000/assr.217 
Arch. de Sc. soc. des Rel., 2002, 118 (avril-juin) 71-84

Maïra MUCHNIK

\title{
NOUVELLES RELIGIOSITÉS ARGENTINES
}

\author{
ORO (Ari Pedro), Axé Mercosul, as religiões \\ afro-brasileiras nos países do prata, Petropolis, \\ Editora Vozes, 1999, 172 p (Préface d'Alejandro \\ Frigerio) (bibliogr., illustr.). \\ FRIGERIO (Alejandro), Cultura Negra en el Cono \\ Sur: Representaciones en Conflicto, Buenos Aires, \\ Ediciones de la Universidad Católica Argentina, 2000, \\ 204 p. (Préface de Robert Farris Thompson) \\ (bibliogr.). \\ CONTEPOMI (María del Rosario), « Nova Era e \\ pós-modernidade: Valores, crenças e práticas no \\ contexto sociocultural contemporâneo » in María Julia \\ CAROZZI (dir.), A Nova Era no Mercosul, Petropolis, \\ Editora Vozes, 1999, 190 p. (bibliogr..). \\ CAROZZI (María Julia), Nueva Era y Terapias \\ Alternativas. Construyendo significados en el discurso \\ y la interacción, Buenos Aires, Ediciones de la \\ Universidad Católica Argentina, 2000, 158 p. \\ (bibliogr..).
}

Si le catholicisme, dans ses ramifications complexes et nombreuses, est l'expression religieuse majoritaire en Argentine, son emprise sociale s'est pourtant relativement affaiblie durant ces quinze dernières années. En effet, à la dictature militaire (1976-1983), caractérisée par l'étroite collaboration entre l'Église et l'État dans la direction du pays, succède, après la période de transition d'Alfonsín, la montée au pouvoir de Carlos Menem. Or, l'époque Menem (1989-1999) est marquée par le déclin accéléré du double monopole de l'Église et de l'État. Non seulement son gouvernement a développé une politique de privatisations touchant de nombreuses entreprises publiques, mais il a modifié également un point important de la Constitution: le Président de la République argentine n'a plus depuis 1994 l'obligation d'être de religion catholique.

Cependant, l'article 2 de la constitution argentine affirmant que " le gouvernement fédéral maintient le culte catholique, apostolique et romain » est toujours en vigueur ; et l'existence du Registre des Cultes Non Catholique du « Secrétariat au Culte » reste un organisme de contrôle efficace envers tous les mouvements reli- 
gieux « dissidents », constituant un frein au respect d'une véritable liberté de culte et du pluralisme en Argentine. Si la religion catholique reste majoritaire on peut pourtant affirmer qu'aujourd'hui, pour une grande partie de la population, l'identité argentine n'est plus synonyme d'identité catholique. L'affaiblissement de son monopole est bien réel, entraînant un fractionnement du champ religieux et une diversification croissante de l'offre religieuse ; ce qui donne la possibilité à de nouveaux acteurs - internes et externes au catholicisme, locaux et étrangers - d'investir cet espace désormais ouvert à la concurrence. C'est notamment le cas de plusieurs mouvements religieux transnationaux dont le développement en Argentine s'inscrit dans la logique de la mondialisation religieuse. Il s'agit entre autres des religions afro-brésiliennes (Umbanda et Batuque), présentes en Argentine depuis les années 1960, et des mouvements New Age nord-américains implantés depuis les années 1980, dont traitent les ouvrages ici présentés.

Le terme de mondialisation englobe une réalité complexe et sous-entend une pluralité de phénomènes comme la diffusion d'un nouveau paradigme technologique, des changements à la fois dans les processus de production, les transactions financières, les marchés du travail, les systèmes d'information et de communication, et dans les modes de consommation, les modes de vie urbains, bref, il signifie un changement des manières de vivre des sociétés et des individus, et il est généralement employé selon une acception large et floue, pour désigner l'une des caractéristiques centrales du changement social que nous vivons à l'entrée dans le troisième millénaire.

Le processus de la mondialisation est caractérisé par l'importance du Marché en tant que régulateur des relations sociales - et pas uniquement économiques - et par un déplacement du centre des attentions de la politique vers l'économie, qui se traduit finalement par un changement du rôle joué par l'État, la figure centrale de l'État-Nation comme unité essentielle de référence s'en trouvant affaiblie.

Tout en nous permettant d'établir comment ces deux caractéristiques du processus de la mondialisation marquent l'organisation et le système de valeurs du New Age et de l'Umbanda/Batuque à Buenos Aires, les textes ici présentés offrent également l'occasion de repenser la mondialisation religieuse proprement dite, en réfléchissant au « processus de décomposition et recomposition des identités individuelles et collectives qui fragilise les limites symboliques des systèmes de croyance et d'appartenance » (E. Pace, "Religião e Globalização », in Globalizaçaõ e Religiaõ, Petrópolis, Vozes, 1997, p. 32).

Ari Pedro Oro retrace les chemins de la diffusion des religions afro-brésiliennes depuis Porto Alegre vers l'Uruguay et l'Argentine, le texte de A. Frigerio contextualise historiquement cette diffusion en "récupérant " la mémoire de la communauté noire de Buenos Aires, et en réhabilitant ainsi un certain multiculturalisme argentin. María Julia Carozzi ainsi que María del Rosario Contepomi étudient le développement des mouvements New Age venus des États-Unis, l'une à Buenos Aires, l'autre à Posadas, capitale de la province argentine de Misiones (Nord-Est argentin) et mettent en évidence les lignes de continuité entre les valeurs, les croyances, les pratiques du New Age et un contexte socioculturel « postmoderne » caractérisé par un individualisme exacerbé. 


\section{I - New Age, Umbanda et Batuque dans le paysage culturel et religieux argentin}

Les auteurs n'ayant pas précisément cherché à inscrire les mouvements religieux dont ils traitent dans le champ religieux argentin global, nous commencerons par ce préliminaire.

Tout d'abord, nous considérerons que ces deux ensembles de pratiques (New Age d'un côté et religions " afro-argentines » de l'autre) ne peuvent être pris séparément mais doivent au contraire être regardés simultanément et dans les relations floues et incertaines pouvant exister entre eux.

Nos auteurs constatent d'une part qu'en Argentine, comme au Brésil, la plupart des temples d'Umbanda et Batuque se situent à la périphérie des grandes agglomé rations, et que les pratiquants, viennent en majorité des classes moyennes, moyennesbasses et basses de la population (Ari Pedro Oro) et d'autre part, que les mouvements New Age concernent avant tout les classes moyennes cultivées des grandes villes, la plupart des centres proposant des ateliers et des cours de tendance New Age à Buenos Aires, étant concentrés dans le quartier de Palermo, au cœur de la Capitale (M. J. Carozzi). Or, il ne faut pas prendre cette division des eaux au pied de la lettre. En effet, Cristián Parker G., dans une étude consacrée aux représentations religieuses dans les quartiers populaires de la banlieue de Santiago de Chile, affirme que sa recherche «montre qu'il existe aussi une influence du New Age dans les secteurs populaires du Grand Santiago, non pas tant de manière organique, mais en tant que religion diffuse " ("Globalizaçã e religião: o caso chileno " in Globalizaçaõ e Religiaõ, op. cit., p.142), sans adopter les pratiques du New Age, les couches populaires de la population chilienne se trouveraient influencées de manière diffuse par le New Age.

Or, nous sommes en mesure d'affirmer que ce constat fait au Chili est également vrai à Buenos Aires, où la plupart des pratiquants de l'Umbanda et du Batuque issus en majorité des couches populaires, ont ou ont eu dans le passé, un intérêt très marqué pour la parapsychologie, que $\mathrm{M}$. J. Carozzi compte au nombre des multiples activités thérapeutiques "alternatives" développées au sein du réseau New Age de Buenos Aires. Nombreux sont les chefs de culte argentins (pères et mères-de-saint des religions afro-brésiliennes) qui accrochent sur les murs de leur temple leur diplôme de parapsychologue, et une large proportion de leurs fidèles ont suivi ou suivent des cours de parapsychologie. L'inverse est vrai également, puisque M. J. Carozzi signale parmi les pratiques New Age les plus populaires à Buenos Aires en 1994, des " adaptations de pratiques religieuses très diverses (thérapie angélique; danse des orixás, magie païenne européenne etc.) ».

Nous sommes donc ici face à une dynamique contemporaine d'interactions entre plusieurs religiosités, que le sociologue argentin Fortunato Mallimaci décrit de manière très expressive dans le passage suivant: "il est possible d'assister à une manifestation catholique de masse comme la Saint Caetano ou de participer à un pèlerinage vers Lujan pour demander pain, travail et santé, tout en lisant quelque chose sur l'énergie positive de quelque auteur nord-américain ou oriental [...] de participer aux cours de yoga ou de contrôle mental de la paroisse ou du club voisin et, bien entendu, d'assister aux cours de préparation pour le baptême d'une 
fille ou d'une filleule... » ("A situação religiosa na Argentina urbana do fim do milênio " in Globalizaçaõ e Religiã̃, op. cit., p. 84). Les emprunts mutuels et diffus entre le New Age d'un côté et l'Umbanda/Batuque de l'autre, semblent bien rendre compte de la logique caractéristique des acteurs religieux argentins contemsymboles " issus d'expériences très diverses.

Inscrire le New Age, le Batuque et l'Umbanda dans le paysage culturel et religieux argentin, implique également de les identifier en tant que pratiques et croyances "alternatives" par rapport à un modèle social dominant. Dans cette perspective, ils occupent une place symboliquement similaire dans le paysage cul-
turel et religieux argentin.

En effet, premièrement, New Age, Umbanda et Batuque sont attachés à des modes de connaissance et d'appréhension de la réalité et du monde opposés aux modèles officiels de la rationalité scientifique. Ainsi, "la critique de la raison et surtout du travail scientifique, focalisée dans le cas du New Age sur une critique de la médecine officielle, et la défense corrélative de formes de connaissance et de techniques curatives anciennes " dont parle Contepomi est entièrement et également applicable aux mouvements magico-religieux " afro-argentins ». Il en va de même pour l'importance de l'écologie et "l'insistance sur le rôle positif de la nature " (Carozzi), dimensions omniprésentes dans l'Umbanda et le Batuque. va de pair avec une organisation concrète de ces mouvements en dehors de toute dynamique véritablement institutionnelle : au sein du New Age il existe, au nom de valeurs individualistes (la réalisation personnelle, le droit à être soi-même, la liberté individuelle de choisir les chemins les plus divers et hétérogènes pour l'auto-épanouissement et la recherche du "moi profond») un " rejet de l'identification et de l'affiliation permanente aux groupes, sociétés et institutions stables et tifs » (Carozzi). Le résme la source possible de problèmes individuels et colleccependant les mêmes tions disparates, dont levues, et d'une multiplicité d'ateliers, de cours, de formad'un festival. Les tentatives de fédéts se réunissent le temps d'une conférence ou Batuque à Buenos Aires, bien que nombreuses différents temples d'Umbanda et de pas dans les temples l'esprit contestataire «ses ont toutes échoué, et s'il ne règne encore moins les valeurs de l'individue "contre culturel " (Carozzi) new ager et tionne indépendamment temple, et accepterait difficiutres. Chaque chef de culte est le seul maître dans son rencontres ne se font qu'au ment une organisation centralisée de sa religion. Les lorsque des Congrès sont organisés.

Enfin, troisièmement, en se référant à l'Orient asiatique (yoga, bouddhisme) et aux indiens nord-américains dans le cas du New Age, ou à l'Afrique dans le cas du Batuque et de l'Umbanda, ces mouvements religieux apparaissent comme culturel" venues de loin » à la fois géographiquementexte argentin. Ce sont des religions 
La prise en compte de ces trois caractéristiques communes nous amène à considérer le New Age, l'Umbanda et le Batuque comme un ensemble de pratiques et de croyances religieuses occupant symboliquement le même espace "alternatif " dans le champ religieux argentin.

\section{II - Différentes formes de circulation religieuse transnationale}

Si nous sommes bien à la fois dans le cas du New Age et dans celui du Batuque/Umbanda, en présence de mouvements religieux transnationaux, force est de constater que cette transnationalisation ne s'opère pas sur le même mode. En effet, si nous tentons de répondre à la question posée par Contepomi - reprenant ici R. L. Segato - sur les rapports qui se tissent entre " exportateurs » et " importateurs ${ }^{\prime}$, nous nous trouvons face à une difficulté flagrante : alors qu'A. P. Oro s'intéresse tout particulièrement à l'analyse de l'élaboration des liens de toutes sortes entre acteurs religieux brésiliens et argentins, M. J. Carozzi est tout à fait laconique sur le sujet. Mis à part quelques brèves allusions à la visite de conférenciers canadiens et nord-américains ou à la traduction dans des revues New Age argentines d'articles parus aux États-Unis, M. J. Carozzi ne s'attarde pas sur les relations transnationales proprement dites. Elle propose de suivre un cadre théorique goffmanien privilégiant l'analyse des micro-structures de l'interaction face à face entre les maitres et les élèves des ateliers et des workshops de la tendance New Age ; la chirurgie des interactions verbales et non verbales construisant minutieusement une nouvelle signification du principe d'autonomie, plus intérieure et plus sacrée, permettant aux new agers d'accéder à une nouvelle perception d'eux-mêmes est brillamment menée par l'auteur.

En revanche, A. P. Oro fait un historique détaillé des liens interpersonnels naissant de la transnationalisation des religions afro-brésiliennes. Il montre clairement qu'elle est le fait d'initiatives absolument individuelles n'obéissant à aucune institution. Étant donnée l'importance des notions de lignage et de famille religieuses dans le monde du Batuque, l'auteur nous livre les noms et le rapide portrait de tous ies chefs religieux de Porto Alegre impliqués dans ce processus. Il prend soin de distinguer les morts ou "pais e mães históricos" selon son expression, des vivants ou "actuels ", au nombre de treize. L'intérêt de son récit est précisément de mettre en avant, au-delà de l'expansion de croyances, de valeurs, de rituels et de marchandises spécifiques, les enjeux de pouvoir qui sous-tendent les échanges entre Brésiliens, Argentins et Uruguayens. Il montre comment le pouvoir charismatique des chefs brésiliens se trouve confirmé et légitimé à travers leurs relations avec les "platinos »; ces derniers imitant les chefs brésiliens à tous points de vue,

${ }^{1}$ « il me paraît impossible de parler d'échanges propres à un monde globalisé, et, dans le cas qui nous occupe, de flux entre communautés religieuses, sans faire une place, dans nos modèles interprétatifs, à cette première division des eaux entre donneurs et récepteurs de modernité, et aux systèmes de circulation de pouvoir et de prestige qui s'établissent entre eux » ("Formações de Diversidade: Naçaõ e Opções Religiosas no Contexto da Globalizaçaõ » in Globalizaçaõ e Religiã̃, Petrópolis, Vozes, 1997). 
dans leurs gestes, leurs postures, leurs discours, ou adoptant pour leurs temples la même architecture et les mêmes symboles religieux. Lorsqu'ils se rendent à Buenos Aires ou à Montevideo, les Brésiliens disent être reçus comme des rois : "Tout le monde fait des efforts pour que je me sente au mieux. Ils m'emmènent diner dans les meilleurs restaurants. Ils sont très dévoués. Quand je vais là-bas, il y a toujours une voiture qui m'attend devant la porte de chez moi ». L'auteur confirme ces témoignages, affirmant que lors de voyages où il accompagna les pais et mães, ces derniers recevaient quantités de cadeaux (bijoux, montres, bagues, alliances, parfums, vaisselle de cristal). Ainsi, il apparaît que les chefs religieux brésiliens qui ont intégré ce réseau d'échange international sont reconnus non seulement pour leur savoir religieux, mais aussi pour leur charisme personnel, ce qui les conforte dans l'idée de détenir un pouvoir légitime sur les "platinos» : " je suis reine dans ma maison et je suis la reine de mon peuple quand j'accomplis mes obligations et quand je vais aux fêtes de mes frères de religion. J'occupe la place qui me revient (...). Montevideo et Buenos Aires sont à mon image, ils ont ma marque » témoigne une mère-de-saint de Porto Alegre.

Si l'appartenance à ce réseau est réservée aux chefs religieux renommés de Porto Alegre, l'initiation par leurs soins, de fils et filles-de-saint étrangers, vient encore accroître leur prestige et leur légitimité au sein de la société brésilienne. Au-delà d'un accroissement de leur capital symbolique sur le marché religieux international (ils sont présidents de fédérations, détenteurs de titres ou se disent les représentants de prestigieux lignages religieux qui, seuls, sont à même de conserver la " pureté » du Batuque) leurs activités au-delà des frontières signifient également un profit économique non négligeable.

A. P. Oro analyse par ailleurs, à partir de témoignages, les représentations de l'Autre circulant entre les différents pays, et la manière dont la construction de ces représentations s'inscrit continuellement au sein des rapports de force et de pouvoir agissant dans ce champ religieux transnational. Ainsi, d'après lui, les pais e mães de Porto Alegre qui ne sont pas parvenus à occuper - ou qui n'occupent plus - une place significative dans les pays du Plata, ceux qui ne sont donc pas à la tête d'un réseau de relations international, sont aussi les pais e mães les plus critiques envers les pratiquants argentins et uruguayens. Selon eux, il est impossible de trouver sur place tous les ingrédients nécessaires à la pratique religieuse (fruits, légumes, herbes variées), il n'y a pas non plus de couturières spécialisées dans la confection des habits rituels, ils déplorent la prolifération du culte aux exus et pombagiras, ainsi qu'une pratique religieuse essentiellement intéressée par l'argent - cette dernière accusation vaut surtout pour les Argentins- les stéréotypes "classiques » sur les Argentins circulant également : ils sont dits "très orgueilleux", "les Argentins sont très arrogants et vaniteux "... Au contraire les pais et mães brésiliens qui ont des fils initiés, ayant leur propre terreiro à Buenos Aires ou à Montevideo sont plus élogieux... De même, du côté des pays de La Plata, les critiques sont plus acerbes envers les chefs religieux de Porto Alegre vivants ou "actuels" qu'envers les "pais históricos», puisque ces derniers, une fois morts, ne sont plus des concurrents redoutables sur le marché religieux afro-brésilien.

Cette différence d'approche appliquée par M. J. Carozzi au New Age et par A. P. Oro aux religions afro-brésiliennes - alors que nous sommes bien dans les deux cas face à des mouvements religieux transnationaux - est sans doute liée, d'une part, au fait que l'expansion de ces deux mouvements ne répond pas à la 
même logique de circulation transnationale. En effet, dans le cas des religions afro-brésiliennes, nous sommes face à une çirculation introduite par des acteurs établissant entre eux des liens étroits autour de la filiation religieuse ; alors que pour le New Age il s'agirait moins d'une circulation de personnes que d'une circulation libre d'idées et de valeurs, c'est-à-dire une transnationalisation donnant lieu à la constitution d'un réseau de relations plus lâches et moins ancrées dans un espace géographique donné.

D'autre part, la divergence entre les approches de M. J. Carozzi et d' A. P. Oro, est liée à une différence fondamentale entre le New Age et les mouvements religieux « afro-argentins » quant à leur réception par la société argentine globale. Si l'« importation" du New Age ne semble en effet provoquer aucune réaction notable, en revanche, les "Afro-Argentins » sont fortement stigmatisés. En d'autres termes, alors que les " Afro-Argentins » sont véritablement perçus comme l'intrusion d'une mouvance étrangère venue d'au-delà des frontières - le thème de "l'invasion des sectes " est rebattu par les media et la presse argentine à propos de l'Umbanda - le New Age semble avoir plus d'affinités avec la culture locale, avec les représentations et les valeurs d'une aspiration à un idéal de modernité circulant parmi une large majorité de la population. L'analyse des formes et des modes d'importation du New Age peut alors sembler moins impérative.

C'est dans le troisième chapitre de son livre qu'A. P. Oro analyse l'image sociale des religions afro-brésiliennes. Il constate que le degré d'acceptation et de légitimité sociales de l'Umbanda et du Batuque est bien supérieur en Uruguay - où elles se développent depuis les années 1950 - qu'en Argentine. A. P. Oro souligne que l'existence dans ce dernier pays du Registre National des Cultes, obligeant les religions afro-brésiliennes - ainsi que d'autres groupes minoritaires comme les Spirites, les témoins de Jéhovah, les Mormons ou les Pentecôtistes - à s'y inscrire, est un signe évident du pouvoir politique du catholicisme argentin qui tente par là même de contrôler et de surveiller ces mouvements religieux non catholiques. Au contraire, en Uruguay, l'Église catholique " ...n'a jamais été à même de diriger un projet effectif de chrétienté » (p. 113). Le pays possède une longue tradition sécularisante, laïque et même anticléricale, construite essentiellement entre les années 1870-1930 (première période de modernisation du pays), qui aboutit à une "décatholisation de l'espace public et à une séparation de l'Eglise et de l'État " (p. 113). Ainsi, la société uruguayenne se montre plus tolérante face aux religions afro-brésiliennes que la société argentine, au sein de laquelle les préjugés et les discriminations sont fortes et évidentes ${ }^{2}$.

L'article d'A. Frigerio "Le candombe argentin: chronique d'une mort annoncée " permet de remonter aux origines historiques de cet état de fait. Cultura Negra en el Cono Sur: Representaciones en Conflicto réunit quatre articles qu'Alejandro Frigerio a publié antérieurement. Seul le premier dont il est question ici, est entièrement consacré à l'Argentine. Les deuxième et troisième portent sur la communauté et la culture afro-uruguayennes, le quatrième analyse les formes d' "art noir " afro-américain et le dernier étudie l'évolution des styles de la capoeira au Brésil. La «chronique d'une mort annoncée " est une chronique socio-historique qui interroge le passé des Noirs en Argentine, à la fois à partir de

${ }^{2}$ On ne peut pas totalement exclure l'idée d'une discrimation liée également à l'appartenance sociale des pratiquants aux couches populaires de la population. 
témoignages recueillis auprès de Noirs argentins contemporains, mais aussi à partir de textes écrits par des chroniqueurs du XIX ${ }^{\mathrm{e}}$ siècle et par les historiens du XX $\mathrm{XX}^{\mathrm{e}}$. La démarche de l'auteur consiste à confronter ces deux sources d'informations dans l'interprétation de l'histoire nationale. En effet, les témoignages des Noirs contemporains signalent qu" " il y a encore seulement quelques années (« et probablement encore de nos jours ") des membres de la communauté afro-argentine jouaient et dansaient une musique qu'ils revendiquaient comme la leur, et qu'ils appelaient candombe".. À partir de ces affirmations, confirmées par d'autres sources (journalistes ayant interviewé des Noirs de Buenos Aires à la fin des années 1960, observateurs étrangers des années 1970) l'auteur établit qu'il existait au moins jusqu'en 1973, à Buenos Aires, une communauté noire se revendiquant comme telle, différenciée d'autres groupes ethniques (des Argentins blancs ou des Noirs uruguayens) dont la principale forme de manifestation était le candombe. Or, ceci vient contredire la position soutenue par la plupart des chroniqueurs et des historiens, pour qui, " la vitalité du candombe en tant que musique, danse et cérémonie folklorique s'est maintenue jusqu'à la chute de Rosas (1852). Il a perdu de son intensité avec la diminution de l'élément afro-argentin et au moment où l'avalanche d'immigrés a «blanchi » la texture ethnographique hétérogène de notre pays... À la fin du siècle dernier, [le candombe] affleurait uniquement pendant les fêtes du carnaval... Nous pouvons considérer la décennie de 1870 comme celle qui marque le début de son inévitable décadence " (Ortiz Oderigo Néstor, Calunga: Croquis del Candombe. Buenos Aires, EUDEBA, 1969, p. 77).

Frigerio s'interroge donc sur les raisons de cette divergence entre les témoignages des uns et des autres: « je souhaiterais maintenant aborder les raisons qui ont amené les spécialistes de la question à ignorer la survie d'une communauté afroargentine aux caractéristiques culturelles propres ». L'auteur considère que ces raisons sont au nombre de deux : premièrement, les Noirs argentins furent pratiquement gommés de l'histoire nationale après 1850 ; deuxièmement, l'utilisation du candombe uruguayen comme modèle, dans la description du candombe argentin " classique ", n'a pas donné de réalité et d'identité aux manifestations culturelles proprement afroargentines. C'est l'un des intérêts majeurs de l'article d'Alejandro Frigerio que de nous fournir une révision bibliographique importante - quoique non exhaustive $^{3}$ - sur l'histoire des Noirs à Buenos Aires. L'auteur suit, pour son argumentation, la piste lancée par Reid Andrews dans The Afro-Argentines of Buenos Aires : 1800-1900 (1980), certainement l'ouvrage le plus complet écrit sur la question jusqu'à présent, où il est dit que « les afroargentins ne sont ni morts ni disparus à aucun moment du siècle passé ; en réalité, ils furent déplacés des registres par les censeurs et les statisticiens, par les écrivains et historiens qui cultivaient le mythe d'une Argentine blanche ". En effet, si le pourcentage d'habitants noirs a considérablement diminué dans la deuxième moitié du XIX ${ }^{\mathrm{e}}$ siècle par rapport à la population totale de l'Argentine, cette diminution n'est pas si importante en chiffres absolus : les "nations " africaines furent remplacées par "les sociétés d'aide mutuelle "; la vitalité de la communauté noire est perceptible à travers la publication de plusieurs journaux communautaires, les répétitions des groupes de carnaval,

3 Nous trouvons d'autres références bibliographiques dans l'ouvrage de Carmen BERNAND, Histoire de Buenos Aires, Paris, Fayard, 1997. 
l'organisation de bals ou encore la création d'associations dont l'une des plus célèbres est le Shimmy Club, fondé en 1922 et qui perdurera jusqu'en $1975^{4}$.

Quant aux danses des Noirs argentins proprement dites, Frigerio regrette qu'aucun des auteurs répertoriés n'en fournisse une description détaillée. Mêmes les études contemporaines sur le Candombe, les "classiques "-soit les références les plus souvent citées sur les Noirs à Buenos Aires - écrits entre les années 1940 et 1980 , reproduisent des descriptions de candombe de Montevideo sans toujours le préciser, contribuant ainsi à la reproduction aveugle d'un candombe stéréotypé qui n'a probablement jamais existé en Argentine, au détriment des manifestations culturelles afro-argentines plus récentes.

Remarquons à ce propos que le candombe, connaît depuis quelques années un véritable renouveau à Buenos Aires, où des dizaines de groupes - composés principalement de jeunes adultes et adolescents - envahissent le dimanche, les places de la capitale, pour répéter face à un public improvisé. A. Frigerio développe actuellement une recherche sur ces jeunes, qui participent à la mise en scène d'une mémoire nationale liée à l'idée de pluralité ethnique argentine. Le recueil « Cultura Negra en el Cono Sur ", dont il est question ici, dans lequel les afro-argentins côtoient les afro-uruguayens et les esclaves noirs du Brésil, en contribuant à défaire le mythe d'une Argentine blanche avant tout européenne, épargnée par le métissage et merveilleusement homogène, s'inscrit donc dans cette tendance à la revalorisation voire à la reconstruction d'un passé argentin pluri-ethnique, suivie par les nouveaux candomberos et par certains pratiquants des religions afro-brésiliennes à Buenos Aires.

\section{III - Les effets de la transnationalisation religieuse}

Nous proposons ici de considérer deux effets majeurs de la transnationalisation religieuse, à savoir premièrement, le reflet de la logique économique mondiale du Marché dans les relations sociales au sein du New Age et des religions afrobrésiliennes en Argentine; deuxièmement, l'affaiblissement de la figure centrale de l'État-Nation comme unité essentielle de référence dans les processus de construction identitaire à l'œuvre à la fois chez les umbandistas/batuqueros et chez les new-agers.

A. P. Oro suggère lui-même que la transnationalisation religieuse dans le Mercosur ne peut être dissociée du contexte régional d'intégration économique croissante dans la mesure où elle est encouragée par l'intensification et la libéralisation des échanges commerciaux caractéristiques de la mondialisation dans son versant économique. Il consacre entièrement le dernier chapitre de son livre aux aspects économiques de la transnationalisation religieuse : "l'accélération de la diffusion des religions afro-brésiliennes vers les pays du Plata dans les années 1980 a accompagné la vague plus générale de la globalisation, certains de leurs

\footnotetext{
${ }^{4}$ Pour les cinq dernières décennies du $\mathrm{XX}^{\mathrm{e}}$ siècle, il existe très peu de sources disponibles.
} 


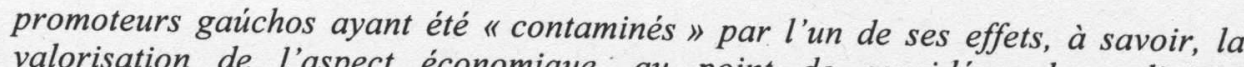
valorisation de l'aspect économique, au point de considérer les religions afro-brésiliennes comme un produit national d'exportation » (p. 153). Il montre premièrement que les va-et-vient des pratiquants et surtout des chefs religieux entre les différents pays, sont liés aux intérêts économiques de chacun. "L'économie, dit-il, même si elle n'est pas le seul élément, peuple l'univers de significations et d'intérêts des acteurs religieux afro-umbandistes, qu'ils soient gaúchos ou platinos » (p. 142). En effet, les pères et mères-de-saint de Porto Alegre pouvaient gagner jusqu'à cinq et six mille dollars en restant cinq jours à l'étranger, soit en initiant de nouveaux fidèles, soit en offrant leurs services à des clients. Leurs propres fils ou filles-de-saint sur place, jouant le rôle d'intermédiaires, leur fournissaient des clients. Les chefs religieux ne semblent pourtant pas s'enrichir personnellement. Suivant davantage la "logique du terreiro" que celle de la société capitaliste environnante, ils réinvestissent la plupart de leur argent dans des activités liées à la religion. Inversement, depuis le début des années 1990, la crise économique dans les pays du Plata a eu pour conséquence de faire diminuer radicalement ces échanges. Dans un deuxième temps. A. P. Oro met en évidence toute l'activité économique qui gravite directement ou indirectement autour de la religion: industries, commerces (vente d'articles religieux: bougies, encens, images, fluides etc.), qui génèrent emplois et circulation de devises.

Sans aborder directement le chapitre de l'économie, M. J. Carozzi dit clairement que la clef du maintien et de la croissance du réseau New Age à Buenos Aires réside dans la recommandation faite aux personnes qui fréquentent les ateliers ou les workshops de circuler le plus possible d'un cours à un autre, d'essayer un maximum d'ateliers. Cette recommandation est justifiée par un discours éthique d'affirmation de l'autonomie individuelle et de la suppression. de l'exercice du pouvoir sur les autres ou des autres sur soi-même (qui suppose donc de ne pas rester « attaché » à un seul professeur, situation pouvant compromettre la liberté de l'individu). Cependant, analysées à la lumière du contexte global de la mondialisation économique, ces recommandations s'apparentent à une sorte de «morale » de la consommation, impliquant concrètement une conduite individuelle de circulation d'une activité à une autre, chacune étant payées au prix «du marché ».

Comme nous le disions plus haut, le processus de la mondialisation est non seulement caractérisé par l'importance du Marché en tant que régulateur des relations sociales mais aussi par un déplacement du centre des attentions de la politique vers l'économie, qui se traduit par l'affaiblissement de la figure centrale de l'État-Nation comme unité essentielle de référence. Or, cette évolution est parfaitement lisible dans les valeurs défendues par le New Age, allant dans le sens d'un " affaiblissement de la notion de projet social global, accompagné d'une tendance à la dépolitisation, à la désyndicalisation » (Contepomi). Ainsi, un new ager de Posadas affirme qu' "il ne peut y avoir de changements dans la société si tu ne changes pas d'abord toi-même. Moi j'ai milité dans la gauche argentine et on était dans l'erreur. Les choses ne s'imposent pas de l'extérieur, le processus doit d'abord se faire à l'intérieur de toi-même » (Contepomi). Le New Age valorise la suppression des hiérarchies établies, et de ce fait, nie explicitement le pouvoir des gouvernements et des gouvernants pour la transformation de l'humanité : "Au-delà des anciens mécanismes du pour et du contre, surgit la dynamique du réveil au côté des autres, dans une zone commune avec ceux qui le pourront et qui le voudront. Indu- 
bitablement, selon la position de la planète et la conjoncture historique, ceci arrivera avec, sans, ou malgré ceux qui sont supposés détenir le pouvoir institutionnel » (propos tenus en 1981 par un leader argentin du mouvement New Age, dans une revue qu'il a lui même fondée) (Carozzi). Ou encore Luis Frejtam, intellectuel new ager argentin, écrit en 1991 dans la revue New Age "Uno Mismo » («Soi même ») fondée à Buenos Aires : « la seule solution aux problèmes de l'humanité, qu'ils soient économiques, politiques ou écologiques, que ce soient des problèmes de violence, de pauvreté ou d'injustice sociale, peut seulement se trouver à l'intérieur de l'être humain [...] Puisque c'est l'esprit humain qui a créé tout ce chaos, c'est seulement là, en le changeant, que l'on peut trouver le chemin vers la sortie [...] Le changement est seulement possible à l'intérieur de nous-mêmes [...] L'injustice sociale naît de ma propre injustice... » (Carozzi).

Quant aux chefs religieux de Porto Alegre responsables de l'"exportation » des religions afro-brésiliennes vers Buenos Aires, ils sont aussi des « activistes culturels » (Oro) qui contribuent, comme par " effet secondaire » à la diffusion de la langue portugaise (considérée comme une langue rituelle sacrée) et à la constitution d'un espace où la référence à une frontière entre des États-Nation séparés perd de sa pertinence. La transnationalisation religieuse signifie ici la construction de nouvelles identités transnationales, l'affirmation d'un sentiment identitaire religieux transcendant les clivages politiques, sociaux et culturels. Les revues, les congrès ou certaines cérémonies religieuses afro-umbandistes réunissent régulièrement des représentants des trois pays (Brésil, Argentine et Uruguay). La transnationalisation afro-brésilienne entraîne la naissance de liens et réseaux de parenté symboliques internationaux à travers la formation d'une «famille-de-saint » étendue aux trois pays. Il n'est pas rare d'entendre des expressions comme " mon frère de Porto Alegre ", "mon frère de Buenos Aires » ou encore "lui, c'est mon frère de Montevideo »(Oro).

Les modes de circulation transnationale différenciés entre un réseau de relations New Age plus distendu et moins " territorialisé " et un ensemble Umbanda/ Batuque organisé et structuré par des relations de filiations religieuses interpersonnelles, donnent lieu à des recompositions identitaires bien distinctes.

Si la mondialisation conduit à l'affirmation d'un sentiment identitaire religieux transcendant les clivages politiques, sociaux et culturels, celle-ci n'annule pas pour autant chez les umbandistes/batuqueros, l'affirmation des particularismes nationaux, "la nationalité et les différences sociales et idéologiques ne sont pas annulées mais superposées à travers la religion » (Oro, p. 93). (Ainsi, dans les congrès « afro-umbandistes » réunissant des représentants des trois pays du Mercosur, chacun affiche son drapeau, symbole nationaliste par excellence). Tout en " décomposant " la référence centrale à l'État-Nation dans la construction identitaire des pratiquants argentins, la transnationalisation des religions afro-brésiliennes entraîne simultanément la recomposition de ce référent à travers la réappropriation d'un passé national africain.

En effet, face aux accusateurs qui les assimilent à des « sectes magiques », les membres des religions afro-brésiliennes mobilisent des stratégies de légitimation qui comportent selon A. Frigerio ${ }^{5}$ - cité par A. P. Oro - deux caractéristiques : à la

${ }^{5}$ A.P. Oro fait ici références aux travaux d'A. Frigerio analysant en profondeur ces processus de légitimation, et non à l'article dont nous traitons dans cette note de lecture. 
fois l'insistance sur la convergence des religions afro-brésiliennes avec les religions monothéistes (en réduisant surtout l'importance des sacrifices d'animaux), et la valorisation de l'africanité du Batuque en tant que tradition religieuse et patrimoine culturel millénaires. De nombreux congrès et colloques publics " afro-umbandistes » ont divulgué ce type de discours afin de faire connaître ces religions et de favoriser leur acceptation sociale. Ce processus, dit de « réafricanisation ", a commencé en Argentine dès les années 1990, à travers le double intérêt pour la langue et la culture du peuple yoruba. À Porto Alegre, et en Uruguay, le mouvement de "réafricanisation" est pratiquement inexistant car "la religion trouve sa légitimation dans son propre passé, elle repose sur une tradition locale propre, elle se reproduit grâce à ses propres racines historiques" (Oro, p. 130). Mais l'Argentine est loin de son passé « noir ». Désireux non seulement de réaliser les rituels mais aussi d'en comprendre le sens, les afro-umbandistes argentins se sont mis à prendre des cours de yoruba, à suivre des séminaires, notamment diffusés par l'Institut de Recherche et de Diffusion des Cultures Noires «Ile Ase Oxum Doyo » fondé par une mère-de-saint renommée de Buenos Aires. Cette quête de l'Afrique passe par une réhabilitation de l'histoire des Noirs d'Argentine : «Les Africains ont un jour fait partie de notre société, et d'une certaine manière, leurs schémas sociaux ont influencé notre propre société" écrit une mère-de-saint (p. 126). La réafricanisation correspond ainsi à la volonté de construire une tradition proprement argentine pour les religions afro-brésiliennes. A. Frigerio affirme que les tenants de l'africanisme à Buenos Aires se considèrent comme les « dépositaires d'une tradition qui représente l'une des racines culturelles argentines fréquemment niée par la culture officielle » (cité pp. 127-128). La mondialisation religieuse facilite ici la (re-)création d'une nouvelle ethnicité, la célébration de la spécificité et de l'altérité ; elle transforme en même temps et radicalement les notions de l'international, du national et du local, comme dans le cas présent d'une "localité globalisée », ou d'une "généralisation de la spécificité » puisque des religions si « particulières » comme les religions afro-américaines - dans le sens où on peut les opposer aux religions dites "universelles » - sont aujourd'hui pratiquées aussi bien au Brésil et dans les Caraïbes qu'en Argentine, aux États-Unis et même - bien que de façon marginale - en Europe.

Dans le cas du New Age, «les nouvelles religiosités, spiritualités ou ésoterismes » contribueraient au contraire à l'affirmation d'identités personnelles et collectives conformes à "l'accomplissement de la logique hégémonique actuelle», en véhiculant des cosmovisions propres à la culture "postmoderne" (Contepomi). L'analyse de María Julia Carozzi, tend à montrer en effet la convergence entre les pratiques, les croyances, les valeurs défendues par le New Age ${ }^{6}$ et celles de la classe moyenne urbaine cultivée - non pas seulement argentine mais occidentale d'une manière générale, l'Occident étant ici entendu comme l'aire d'influence pri-

${ }^{6}$ Les disciplines les plus répandues dans le circuit alternatif de Buenos Aires sont très nombreuses, elles vont des thérapies naturelles (thérapie florale, hydrothérapie, feng shui), psychothérapies non analytiques (thérapie bioénergétique, diverses psychothérapies corporelles, thérapie du jeu, programmation neurolinguistique, ontologie du langage) et médecines alternatives ou d'origine orientale (homéopathie, acupuncture, shiatzu,etc,) aux disciplines ésotériques (astrologie, numérologie, graphologie, tarot), en passant par des techniques du mouvement orientales ou alternatives à la gymnastique et à la danse occidentales (tai-chi-chuan, yoga, technique Alexander, biodanse...) et des techniques de méditation plus ou moins inspirées du bouddhisme, du yoga, du mysticisme chrétien, ou des indigènes nord-américains (sweat lodges et chaminisme). 
vilégiée du modèle de vie américain - héritière et dépositaire des idéaux de paix et de liberté des années 1970. Ainsi, le rejet de l'identification et de l'affiliation permanente aux groupes, sociétés et institutions stables et durables serait l'approfondissement de formes de sociabilité propres aux classes moyennes urbaines instruites de l'Occident d'aujourd'hui, à savoir « l'acceptation de la polygamie successive, ou les programmes d'études universitaires permettant la sélection et la combinaison individuelle des matières, la libéralisation des lois régulatrices de l'emploi, aboutissant tant dans la famille, qu'au travail et dans l'éducation, à des liens toujours fugaces et changeants " ; la valorisation de la suppression des hiérarchies de pouvoir établies, ou leur inversion est mise en rapport par l'auteur avec de nombreux mouvements sociaux post-années soixante, qu'il s'agisse du nouveau pacifisme, du nouveau féminisme ou du mouvement écologique. Le mouvement New Age s'inscrirait alors au sein d'un «macro-mouvement antihiérarchique dont les protagonistes sont issus du secteur le plus éduqué parmi ceux qui étaient jeunes dans les années 1960 ». L'insistance sur le rôle positif de la nature serait l'approfondissement d'une tendance propre à d'autres mouvements sociaux post-années soixante, opposés à la domination technologique aveugle de la nature, comme les mouvements antinucléaire, écologique ou écoféministe répandus parmi les classes moyennes urbaines et instruites à partir des années 1960. Enfin, l'affirmation de l'autonomie individuelle correspond à «l'approfondissement d'une valeur de la culture nord-américaine qui, à travers la diffusion de la technologie et des produits culturels en provenance des États-Unis, s'est répandue bien au-delà des limites de ce pays et même de l'Occident ", un approfondissement qui va jusqu'à ériger le principe d'autonomie en religion, selon la remarquable analyse de M. J. Carozzi. Il est rare, dit l'auteur, en Argentine comme aux États-Unis, que quelqu'un se reconnaisse comme un new ager ou comme faisant partie du mouvement. Cette absence d'identification stable à un groupe, évitant toute inscription sociale de l'interlocuteur et entraînant un type de discours universalisant (référence à Dieu ou à l'énergie universelle), permet au professionnel du réseau New Age de se nier soi-même en tant qu'être socialement déterminé, et d'affirmer par conséquent sa totale autonomie personnelle (pas d'influences extérieures et pas d'intention d'influencer les autres). Corrélativement, la coïncidence amenant des personnes différentes à l'adoption de pratiques et de croyances similaires se reproduisant au sein du réseau New Age, est exposée par les membres actifs du mouvement comme la conséquence de faits surnaturels, d' " énergies qui vibrent à l'unisson ", d' " interconnexions énergétiques ou de la « synchronisation jungienne ». Selon María Julia Carozzi, cette logique serait poussée à l'extrême parmi les classes moyennes urbaines occidentales du New Age, pour qui « le principe d'autonomie vis-à-vis de tout conditionnement socioculturel devient un quelque chose, ayant une existence propre et une capacité d'action : le moi supérieur, le guide, le maître, le guérisseur, le chaman intérieur [...] L'autonomie individuelle devient ici sujet. [...]» et «cet intérieur parfait » n'est pas seulement réifié mais aussi sacralisé (« étincelle divine intérieure »), l'autonomie est donc, d'après l'auteur, conçue comme divine. Le New Age fait de l'autonomie sa religion.

Au terme de cette présentation, il apparaît donc que l'analyse de la modernité argentine, caractérisée entre autres par une diversification culturelle et religieuse croissante, ne peut faire l'économie d'une approche par le biais de la transnationalisation ou mondialisation. Ce processus de mondialisation implique, comme nous 
venons de le voir, une double dynamique, à la fois d'homogénéisation et de différenciation locale. En véhiculant les valeurs et le modèle hégémonique du mode de vie nord-américain, le New Age tendrait à favoriser une définition homogénéisante de la mondialisation. En revanche, le développement du Batuque et de l'Umbanda, permettant à la fois la revendication d'une spécificité de l'histoire nationale argentine, et la construction de nouvelles identités transnationales ancrées dans la région du Mercosur, va plutôt dans le sens d'une résistance du local envers le global. Cependant, l'accent mis sur l'un ou l'autre versant du processus de mondialisation ne serait-il pas une question de point de vue ? En effet, à l'inverse ne serait-il pas pertinent de relever les mécanismes proprement locaux d'appropriation des croyances, des pratiques et des valeurs New $\mathrm{Age}^{7}$, ou de mettre en rapport la redécouverte argentine de la pluralité ethnique avec le principe du multiculturalisme cher à la modernité nord-américaine?

Il reste que nous sommes aujourd'hui en Argentine dans une situation de changement social, économique et politique très rapide, rapidité qui en déstabilisant la population et en provoquant l'incertitude générale, pourrait bien activer la prolifération des croyances. Là où la confiance dans la capacité du politique à diriger la nation a disparu, où l'économie est en sursis et où la population angoissée ou affamée prend d'assaut les supermarchés comme en décembre dernier, la confusion et la perte de repères désormais installés sont des facteurs possiblement favorables à une redistribution des modalités du croire et à une recomposition significative du
paysage religieux.

\author{
Maïra MUCHNIK \\ Centre d'Études Interdisciplinaires des Faits Religieux
}

\footnotetext{
${ }^{7}$ Dans le cas de Posadas en particulier décrit par Contepomi, sachant que la province de Misiones fait partie de l'ancienne région guarani, et que cette culture indienne marque encore clairement le payculture locale et la culture New Age. Nous n'ave intéressant de mieux définir les pont éventuels entre la ment un certain succès à Buenos Aires, mais lens pas parlé ici des pentecôtistes qui connaissent égalethème de « la réinvention du diable dans l'Égle travail de l'anthropologue argentin Pablo Semán sur le un bon contre-exemple, car il met précisément en évidenelle du Royaume de Dieu à Buenos Aires » est et des rituels qui s'imposent aux pasteurs de l'Église Universelle daptations et modifications du discours culturelles de l'affliction, de la souffrance et du mal diffèrent de dans un contexte où les représentations par l'Église au Brésil.
} 